\title{
Particle Dynamics at the Onset of the Granular Gas-Liquid Transition
}

\author{
M. Noirhomme $\odot,{ }^{1,}{ }^{*}$ A. Cazaubiel, ${ }^{2}$ E. Falcon $\odot,{ }^{2, \dagger}$ D. Fischer $\odot,{ }^{3}$ Y. Garrabos,${ }^{4}$ C. Lecoutre-Chabot, ${ }^{4}$ \\ S. Mawet $\odot,{ }^{1}$ E. Opsomer $\odot,{ }^{1}$ F. Palencia $\odot,{ }^{4}$ S. Pillitteri, ${ }^{1}$ and N. Vandewalle $\odot^{1, \#}$ \\ ${ }^{1}$ GRASP, CESAM Research Unit, Institut de Physique B5a, Sart Tilman, University of Liège, B-4000 Liège, Belgium \\ ${ }^{2}$ Université de Paris, Matière et Systèmes Complexes (MSC), UMR 7057 CNRS, F-75013 Paris, France \\ ${ }^{3}$ Institute of Physics, Otto von Guericke University, D-39106 Magdeburg, Germany \\ ${ }^{4}$ CNRS, Université de Bordeaux, Bordeaux INP, ICMCB, UMR 5026, F-33600 Pessac, France
}

(Received 2 October 2020; accepted 4 March 2021; published 26 March 2021)

\begin{abstract}
We study experimentally the dynamical behavior of few large tracer particles placed in a quasi-2D granular "gas" made of many small beads in a low-gravity environment. Multiple inelastic collisions transfer momentum from the uniaxially driven gas to the tracers whose velocity distributions are studied through particle tracking. Analyzing these distributions for an increasing system density reveals that translational energy equipartition is reached at the onset of the gas-liquid granular transition corresponding to the emergence of local clusters. The dynamics of a few tracer particles thus appears as a simple and accurate tool to detect this transition. A model is proposed for describing accurately the formation of local heterogeneities.
\end{abstract}

DOI: 10.1103/PhysRevLett.126.128002

Granular gases are composed by a large amount of mobile solid particles. Unlike molecular gases, they are characterized by inelastic collisions leading to an ongoing loss of kinetic energy over time [1-4]. In order to avoid such cooling of the system, mechanical energy can be injected in the granular gas. During the two past decades, it has been shown that driven granular gases can exhibit different dynamics depending on the nature of their driving mechanism. For boundary-driven system (e.g., mechanically shaking), several laws have been proposed to describe their velocity distributions [5-8] but most of them correspond to non-Gaussian distributions with densitydependent stretched exponential tails [8-11]. Nonequipartition of kinetic energy between degrees of freedom is also reported [12-14]. Recent experimental studies using a spatially homogeneous forcing in volume (by magnetic driving of each particle) leads to several major differences such as velocity distributions with density-independent exponential tails [15].

From dilute systems, increasing the number of particles also leads to the spontaneous emergence of denser regions (called "granular liquid droplets") coexisting with the granular gas. The gas-liquid transition is known to depend on the collision rate in the system [16-21]. Although this phenomenon has already been studied at the scale of the entire system, local clustering is still poorly investigated [22-24]. The granular gas-liquid transition itself is smooth and many fundamental questions remain open. Among others, the particle dynamics (in the gas phase) near the transition is still unclear. Because of the nucleating local heterogeneities at the transition, particle tracking becomes hard to implement in particular for 3D systems. Indeed, the experimental data on granular materials generally consist of images of the studied system. The overlaps between particles make their tracking particularly complicated. While machine learning methods can help in sufficiently diluted systems [25], 3D tracking is not possible in denser systems, simply based on the images of the experiment. Experimental tricks should therefore be used.

In this Letter, we investigate the gaslike to clustering transition in driven granular media through the tracking of a few large tracer particles in a 2D gas made of semitransparent tiny particles. We show that the velocity distributions of these tracers show peculiar features when the density of the system is increased above some threshold value. The latter is shown to coincide with the emergence of clusters, that is a liquidlike phase coexisting with the granular gas.

Even though granular gases can be created on Earth by using strong agitation [26,27], a precise investigation of their dynamics requires the absence of gravity. Our studies were thus realized using the VIP-Gran instrument [28] that was developed in the frame of the SpaceGrains project [29] of the European Space Agency (ESA). Experiments took place during the 66th and 69th ESA parabolic flight campaigns, organized by Novespace in Mérignac, France. Given our need to achieve particle tracking, granular media is enclosed in a quasi-2D cell composed of four transparent walls and two moving plates that can inject kinetic energy into the system. The container is characterized by its average length $L=50 \mathrm{~mm}$, its width $l=30 \mathrm{~mm}$, and its height $h=5 \mathrm{~mm}$. Pistons move sinusoidally in phase opposition along the $x$ axis with fixed amplitude $A=4 \mathrm{~mm}$ and angular frequency $\omega=2 \pi f$, with $f=15 \mathrm{~Hz}$. 
The system is filled with $N_{t}$ large aluminum beads of diameter $D=3 \mathrm{~mm}$ used as the tracers and $N_{s}$ small glass beads of diameter $d=1 \mathrm{~mm}$. The volumes of both particle species are denoted $V_{t}$ for the large tracers and $V_{s}$ for the small beads. Tracers are placed in the container before takeoff while small particles are injected into the cell during the flight using a bead-feeder device [28]. Each experimental run is started after entering zero gravity conditions lasting $20 \mathrm{~s}$. Starting without small beads, additional particles are injected after every fifth parabola so that 7 different diluted fillings were explored: $N_{s} \in\{0 ; 50 ; 100 ; 150 ; 200 ; 250 ; 300\}$. The mean packing fraction is thus $\langle\phi\rangle \equiv \pi\left(N_{s} d^{2}+N_{t} D^{2}\right) /(4 L l)$ that ranges from 0 to $17.1 \%$. Experiments corresponding to the same filling have been repeated 5 times, on the one hand, to increase the statistics, and on the other hand, to average the effect of the $g$ jitters [28]. Raw data are collected using a high-speed camera with a frame rate of $900 \mathrm{fps}$. Assuming that the particle rms velocity is $A \omega \simeq 0.38 \mathrm{~m} / \mathrm{s}$, this frame rate is sufficient for reliable particle tracking [30]. The almost $1 \times 10^{6}$ images thus produced were processed using the open access software FIJI [31].

Snapshots of the experiments are given in Fig. 1. One can see that trajectories differ when the number of small particles increases. For dilute cases, tracers are struck by the pistons and exhibit ballistic motion along the $x$ direction. However, when the collision rate with the surrounding particles increases, tracers no longer interact with the pistons and remain in the center of the cell. Some erratic motion, reminiscent of a $2 \mathrm{D}$ random walk, is reported along both $x$ and $y$ directions. Using an optical method [32], we extract the mean density profiles in the direction of vibration. Particles are nearly uniformly distributed at low fillings (for $N_{s}<200$ ). For $N_{s} \gtrsim 200$, the distributions are more peaked, indicating a denser zone in the middle of the system.

By tracking the tracers over time, we can compute their velocity distribution $v_{x}$ and $v_{y}$ along the $x$ and $y$ axes. Corresponding probability density functions (PDFs) for a single tracer $\left(N_{t}=1\right)$ are plotted in Figs. 2(a) and 2(b) using nondimensional velocities $v_{i} /(A \omega)$. For dilute situations, the PDFs are quite different along both axes (see the black squares). Along the $x$ axis, one notes a large dispersion of the central peak when $N_{s}=0$. This is characteristic for a regime in which the tracer alternatively strikes both pistons and thus bounces back and forth through the cell. These round-trips become less frequent as $N_{s}$ is increased. High velocities are less likely and the fat tails of the distributions progressively disappear until $N_{s}=200$. Beyond this critical value [see green, light blue, and dark blue curves in Figs. 2(a) and 2(b)], higher velocities become more frequent again. Looking at the PDF along the $y$ axis, one sees that PDFs are similar exponential distributions for $N_{s} \lesssim 200$ but then widen dramatically once this threshold is exceeded. PDFs become stretched exponential distributions [see light blue and dark blue curves in Fig. 2(b)].

In order to emphasize these considerations, we plot in Fig. 2(c) the standard deviations $\sigma=\sqrt{\left\langle v^{2}\right\rangle_{t}}$ of the tracer velocity distributions, and its excess kurtosis $\kappa=\left\langle v^{4}\right\rangle_{t} / \sigma^{4}-3$, as a function of $N_{s}$ for the vibrating and normal directions (respectively noted $\sigma_{x}, \sigma_{y}, \kappa_{x}$, and $\kappa_{y}$ ). The standard deviation is an indicator of the width of the distribution while the excess kurtosis is linked to the heaviness of their tails $(\kappa=0$ corresponds to a normal distribution). Note that error bars are not visible on these graphs because of their small values. As the number of gas particles increases, $\sigma_{x}$ becomes smaller and $\kappa_{x}$ increases until $N_{s}=200$. It means that less probable large fluctuations of rms velocities are observed corresponding to fewer and fewer interactions with the pistons. For denser systems, $\sigma_{x}$ tends to the value of $\sigma_{y}$. This means that the kinetic energy of the tracers is now equipartitioned. As revealed by the PDF, the rms tracer velocity (and therefore the rms fluctuations of their kinetic energy) reaches its minimum at $N_{s}=200$. Above this value, the width of all distributions increases. In addition, higher rms velocities with less probable large fluctuations are observed, as evidenced
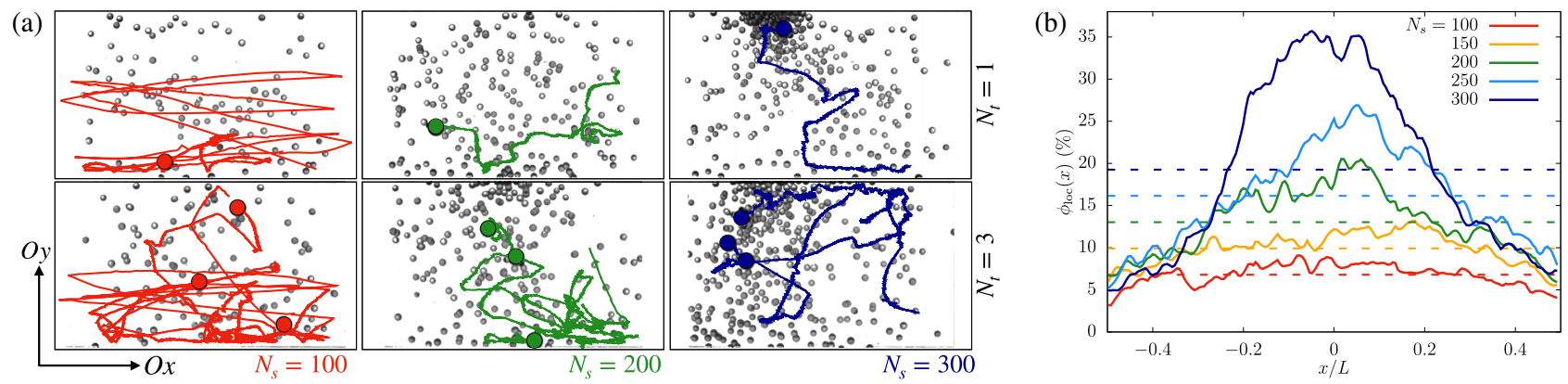

FIG. 1. (a) Pictures taken from the bottom of the cell for three different fillings in small beads $N_{s}=100$ (red), $N_{s}=200$ (green), and $N_{s}=300$ (blue) and two different numbers of tracers, $N_{t}=1$ (left) and $N_{t}=3$ (right). The full trajectories of the tracers are drawn in colors. Vibrations are along the $x$ axis. At $N_{s}=300$, a granular liquid droplet is observed. (b) Typical mean density profiles obtained in the $x$ direction, at variable $N_{s}$ and fixed $N_{t}=1$. Uniform distributions are depicted by the dashed lines and $x=0$ corresponds to the middle of the cell. 

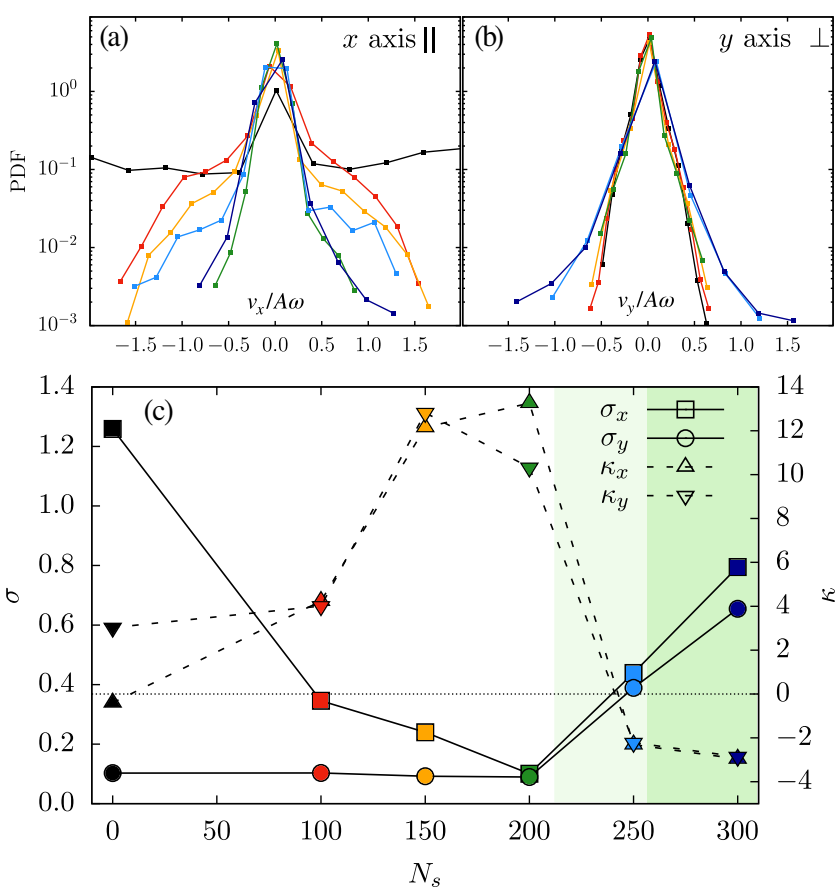

FIG. 2. PDFs of the measured velocities of the tracer along the (a) $x$ axis parallel to excitation and (b) the $y$ axis. The different colors are linked to the different fillings in small beads: $N_{s} \in\{0 ; 100 ; 150 ; 200 ; 250 ; 300\}$. (c) Standard deviations $\sigma_{x}$ and $\sigma_{y}$ (left axis) and excess kurtosis $\kappa_{x}$ and $\kappa_{y}$ (right axis) of the above velocity distributions, as a function of $N_{s}$. For $N_{s} \lesssim 200, \sigma_{x}$ and $\sigma_{y}$ are different, meaning that the velocity distributions are different in the two perpendicular directions $x$ and $y$, as expected. At $N_{s} \approx 200, \sigma_{x}$ reaches its minimal value, and the distributions are as peaked as possible. For $N_{s} \gtrsim 200$, the standard deviations over the $x$ and $y$ axis collapse and the value of the excess kurtosis decreases dramatically.

by the increase of $\sigma_{x}$ and $\sigma_{y}$ and the dramatic decrease of $\kappa_{x}$ and $\kappa_{y}$, both being negative. The PDFs undergo a transition at $N_{s} \approx 200$, going from a platikurtic profile to a leptokurtic one. This is counterintuitive since the number of collisions is expected to increase with the packing fraction of the system. So, is this behavior the signature of the emergence of particle clustering?

In order to answer this question, we divide the cell into squared subsystems of side $c=10 \mathrm{~mm}$ [see inset of Fig. 3(a)] and consider that two phases (gaseous and liquid) can coexist. We measure the number of times that the 2D local density $\phi_{\text {loc }}$ is found in these subsystems, its occurrence frequency being denoted $F\left(\phi_{\text {loc }}\right)$. It has been previously shown that phase transitions can accurately be predicted with the help of this method [33,34]. The value of $\phi_{\text {loc }}$ is therefore between 0 (no particle) and 1 (subsystem fully opaque). This maximum value can be reached because of possible overlaps between particles. Finally, in order to compare the local density distributions with each other, they are normalized by the global fraction of the particles in the quasi-2D system $\langle\phi\rangle$.
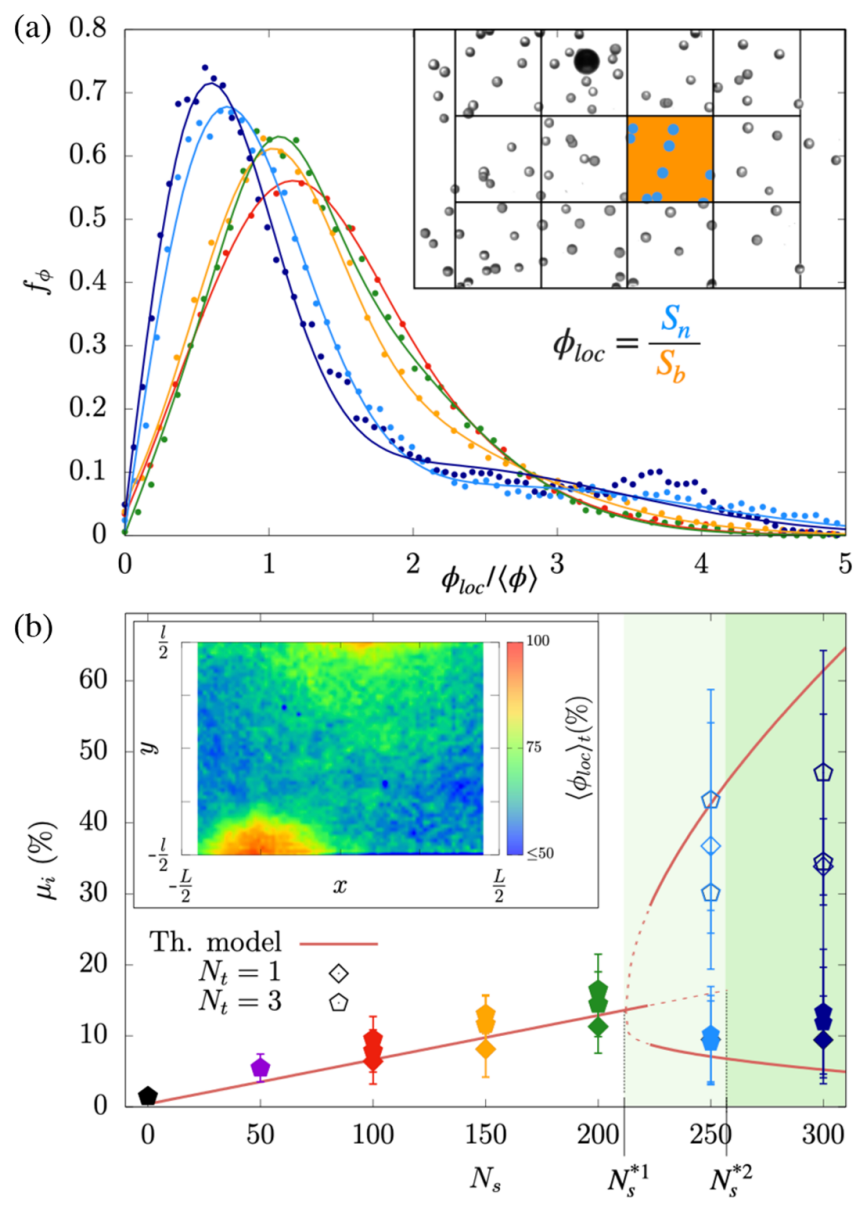

FIG. 3. (a) Distribution of normalized 2D local density $\phi_{\text {loc }} /\langle\phi\rangle$ of small beads for 5 different $N_{s}$ (same colors as in Fig. 2). One or two maxima are observed in the distributions, indicating the presence of one or two phases in the system. Inset: the 2D local density is measured in a square of side $c$. (b) Mean 2D local density $\mu_{i}=\mu_{g}$ or $\mu_{l}$ (in \%) inferred by fitting the distributions in (a) with Eq. (1). For $N_{s} \leq N_{s}^{* 1}, \mu_{g}=\mu_{l}=\langle\phi\rangle$, as expected for uniformly distributed particles. For $N_{s}^{* 1} \leq N_{s} \leq N_{s}^{* 2}$, a bifurcation takes place. The particles can either stay gaseous or gather in a fluid droplet. For $N_{s} \geq N_{s}^{* 2}$, the system is only stable when a droplet has nucleated. This can be predicted by a theoretical model (red curves; see main text). Inset: local density $\left\langle\phi_{\text {loc }}\right\rangle_{t}$, averaged over time, showing the droplet nucleation at the "cold" side walls $\left(N_{s}=300\right)$.

The normalized distributions $f_{\phi}=F\left(\phi_{\text {loc }} /\langle\phi\rangle\right)$ are shown as a function of $\phi_{\text {loc }} /\langle\phi\rangle$ in Fig. 3(a) for all $N_{s}$. As shown in this figure, one master curve can be distinguished. Indeed, for $N_{s} \lesssim 200$, all $f_{\phi}$ collapse onto the same curve that presents one single maximum at $\phi_{\mathrm{loc}} \approx\langle\phi\rangle$. According to Refs. [33,34], this means that the system is in a gaslike state. On the contrary, for higher fillings $\left(N_{s} \gtrsim 250\right)$, the distributions exhibit a second maximum, localized at $\phi_{\mathrm{loc}} \sim 3.5\langle\phi\rangle$, meaning that a liquidlike phase emerged in the cell. At the same time, the position of the average gas density is shifted toward $\phi_{\mathrm{loc}} \approx 0.7\langle\phi\rangle$, meaning that the gas phase is diluted. Note that our results do not 
depend on the chosen value of the cell size in the range $c \in[5,15] \mathrm{mm}$.

One notices that the velocity distributions of the tracers in Figs. 2(a) and 2(b) are directly linked to the states of the entire system [Fig. 3(a)]. At low packing fractions, tracers simply bathe in a homogeneously distributed granular gas. Maintaining homogeneity and increasing the number of particles implies unavoidably an increase in the collision rate and therefore a decrease in the kinetic energy of tracers. The dissipative character of the collisions in the gas adds another ingredient to the problem: particles are able to condense spontaneously, if the collision rate is high enough [19-21,32]. For $N_{s} \gtrsim 200$, the frequently observed high local densities are due to the episodic trapping of tracers in local clusters as seen in Fig. 1(a). Indeed, if the collision rate is sufficiently high, the density around a tracer can increase [near 3.5 $\langle\phi\rangle$ in Fig. 2(a)] and yields in the local formation of a cluster. In addition, once formed, the cluster exhibits a semicylindrical shape, as evidenced in the inset of Fig. 3(b), where the smallest possible subsystems $(2 \times 2$ pixels $)$ are colored as a function of the time averaged local densities. As a result of the formation of the cluster, the density of the rest of the system decreases $\sim 0.7\langle\phi\rangle$. If a tracer escapes from such a dense neighborhood, it suddenly finds itself in a very dilute environment and gains more mobility. Successions of trapping and cluster evasion explain thus the widening of tracer velocities. Therefore, analyzing these velocities gives access to the state of the entire system. The tracer is ultimately no longer a simple particle that can be tracked but a marker making it possible to infer whether a liquid phase coexists within a granular gas.

Finally, we adjusted the measured local density distributions $f_{\phi}=x_{g} f_{g}+x_{l} f_{l}$ by gas and liquid components, $f_{g}$ and $f_{l}$, assuming

$$
\begin{gathered}
f_{g}=\frac{1}{\mu_{g}^{2}} \frac{\phi_{\mathrm{loc}}}{\langle\phi\rangle} \exp \left[-\frac{1}{2 \mu_{g}^{2}}\left(\frac{\phi_{\mathrm{loc}}}{\langle\phi\rangle}\right)^{2}\right], \\
f_{l}=\frac{1}{\sigma_{l} \sqrt{2 \pi}} \exp \left[-\frac{\left(\phi_{\mathrm{loc}} /\langle\phi\rangle-\mu_{l}\right)^{2}}{2 \sigma_{l}^{2}}\right],
\end{gathered}
$$

where $\mu_{g}$ and $\mu_{l}$ are mean 2D local densities and $x_{g}$ and $x_{l}$ denote, respectively, the volume fractions of the gas and the liquid phases. The fractions of the gas and the liquid phases obey $x_{g}+x_{l}=1$. We have chosen $f_{l}$ as Gaussian distribution, as in Ref. [34], where the simulated system is periodic. This is reasonable for the liquid phase, but our choice has been empirically oriented toward a Rayleigh distribution for $f_{g}$. Indeed, this asymmetric distribution corresponds better to our bounded system. The result of the fits is plotted in Fig. 3(a) and the extracted values of $\mu_{g}$ and $\mu_{l}$ are shown in Fig. 3(b), as a function of $N_{s}$, evidencing a pitchfork bifurcation shown in red.
Let us consider that the system reached a stationary state. Every period $T$, a kinetic energy $\left\langle E_{i}\right\rangle=n_{g} k_{i}$ is given to the $n_{g}$ grains in the vicinity of a piston. The resulting energy wave is dissipated through the system via several inelastic collisions and the remaining energy $\left\langle E_{r}\right\rangle$ reaches the other cell side after a typical time $\tau>T$ [21]. Therefore, there are a number of waves $n_{w}=\tau / T$ that are dissipated in the system during $T$, otherwise the system cannot remain in a stationary state. Our goal is to apply Onsager's variational principle $[35,36]$ according to which a system evolves toward a stationary state with the least possible dissipation. The dissipated energy is equal to $\left\langle E_{D}\right\rangle=\left\langle E_{i}\right\rangle-n_{w}\left\langle E_{r}\right\rangle$. Let us consider that the $N_{s}$ particles can be eventually divided into two populations: $N_{g}$ beads belonging to the gaseous phase and $N_{s}-N_{g}$ to the liquid phase. Moreover, one can express $\left\langle E_{r}\right\rangle$ as a function of $\left\langle E_{i}\right\rangle$ and $\bar{n}_{\text {col }}$, the number of collisions suffered by each energy wave during $T$ [21]. Finally, if a liquid droplet has formed in the system, the energy wave is partially lost in it, so that $\left\langle E_{r}\right\rangle=\left(1-p_{l}\right)\left\langle E_{i}\right\rangle \varepsilon^{2 \bar{n}_{\mathrm{col}}}$, where $p_{l}$ is the proportion of lost energy. Accordingly, one can define

$$
\left\langle E_{D}\right\rangle=n_{g} k_{i}\left(1-\frac{\tau}{T}\left(1-p_{l}\right) \varepsilon^{2 \bar{n}_{\mathrm{col}}}\right),
$$

where $n_{g}=4 A l \mu_{g} /\left(\pi d^{2}\right), \quad p_{l}=d / l \sqrt{\left(N_{s}-N_{g}\right) /\left(2 \mu_{l}\right)}$, $\bar{n}_{\text {col }}=8 \pi A \mu_{g} / h, \tau / T=\left\{\left[h /\left(4 \mu_{g}\right)-d\right] / 2 \pi A\right\}\left\{\left[1-\varepsilon^{-4 \mu_{g} L / h}\right] /\right.$ $\left.\left(1-\varepsilon^{-1}\right)\right\}[21,37]$, and surface and mass conservation in the system yield the following relationship between $\mu_{g}$ and $\mu_{l}$ :

$$
\mu_{l}=\frac{\pi d^{2}\left(N_{s}-N_{g}\right) \mu_{g}}{4 \mu_{g} L l-\pi d^{2} N_{g}} .
$$

Further details are given in Ref. [37]. Given Eqs. (2) and (3), one shows that the mean dissipated energy depends on the number of gaseous particles $N_{g} \in\left[0 ; N_{s}\right]$. Following Onsager's principle, we determine numerically the number of gas particles $N_{g 0}$ that minimize Eq. (2) while verifying Eq. (3) for all values of $N_{s}$. Obviously, $N_{g 0}$ also gives access to the number of particles in the liquid phase, and the volume of the latter via Eq. (3). The red curve in Fig. 3(b) gives the packing fraction of both phases as a function of $N_{s}$. Either there is one single gaseous phase in the system (see the straight line corresponding to $N_{g 0}=N_{s}$ ) or a droplet is formed (see the two other curves). One can see that our model is able to predict accurately the mean densities of both phases as well as the critical number of particles $N_{s}^{* 1} \approx 212$ triggering the gas-liquid transition, being a bifurcation similar to the one observed by Herminghaus and co-workers [38,39]. It is interesting to note that the mean dissipated energy exhibits two minima in the range $N_{g} \in\left[N_{s}^{* 1} ; N_{s}^{* 2}\right]\left(N_{s}^{* 2} \approx 255\right)$. The global (local) minima of $\left\langle E_{D}\right\rangle$ give the most (less) stable mean 
densities of both phases, which are depicted by the plain (dashed) red curves in Fig. 3(b).

To conclude, we investigated the dynamics of large tracer particles placed in a mechanically excited granular gas. System density plays a crucial role in the observed dynamics. Indeed, above some threshold filling, tracers experience successive episodes of local trapping yielding in large mobility fluctuations. Particle tracking has shown that the velocity distributions of the tracers are strongly linked to the state of the system and can be used to infer whether a liquid phase coexists within a granular gas. Finally, we developed a theoretical model based on Onsager's minimum rate of dissipation principle, that fits our observations.

We thank M. Braibanti and V. Koehne from ESA as well as R. Stannarius from Otto von Guericke University for fruitful discussions. VIP-Gran-PF instrument was built by DTM Technologies (Modena, Italy). This work was funded by European Space Agency Topical Team SpaceGrains No. 4000103461. We thank the support of Novespace during ESA Parabolic Flight Campaigns. M. N. thanks the Belgian Federal Science Policy Office (BELSPO) for the provision of financial support in the framework of the PRODEX Programme of the European Space Agency (ESA) under Contract No. 4000103267. E. F., Y. G., C. L.-C., and F. P. thank CNES for partial financial support. D. F. acknowledges funding by DLR within project EQUIPAGE II (50WM1842).

*mnoirhomme@uliege.be

† eric.falcon@univ-paris-diderot.fr

*nvandewalle@uliege.be

[1] C. C. Maßß, N. Isert, G. Maret, and C. M. Aegerter, Phys. Rev. Lett. 100, 248001 (2008).

[2] Y. Grasselli, G. Bossis, and G. Goutallier, Europhys. Lett. 86, 60007 (2009).

[3] S. Tatsumi, Y. Murayama, H. Hayakawa, and M. Sano, J. Fluid Mech. 641, 521 (2009).

[4] K. Harth, T. Trittel, S. Wegner, and R. Stannarius, Phys. Rev. Lett. 120, 214301 (2018).

[5] T. P. C. van Noije and M. H. Hernst, Granular Matter 1, 57 (1998).

[6] A. Puglisi, V. Loreto, U. Marini Bettolo Marconi, A. Petri, and A. Vulpiani, Phys. Rev. Lett. 81, 3848 (1998).

[7] C. Scholz and T. Pöschel, Phys. Rev. Lett. 118, 198003 (2017).

[8] F. Rouyer and N. Menon, Phys. Rev. Lett. 85, 3676 (2000).

[9] W. Losert, D. G. W. Cooper, J. Delour, A. Kudrolli, and J. P. Gollub, Chaos 9, 682 (1999).

[10] J. S. Olafsen and J. S. Urbach, Phys. Rev. E 60, R2468(R) (1999); A. Kudrolli and J. Henry, Phys. Rev. E 62, R1489 (2000); J. S. van Zon, J. Kreft, D. I. Goldman, D. Miracle, J. B. Swift, and H. L. Swinney, Phys. Rev. E 70, 040301(R) (2004); H.-Q. Wang, K. Feitosa, and N. Menon, Phys. Rev. E 80, 060304(R) (2009); Y. Chen, M. Hou, Y. Jiang, and M. Liu, Phys. Rev. E 88, 052204 (2013).

[11] T. Kanzaki, R. C. Hidalgo, D. Maza, and I. Pagonabarraga, J. Stat. Mech. (2010) P06020.
[12] H.-Q. Wang and N. Menon, Phys. Rev. Lett. 100, 158001 (2008).

[13] K. Harth, U. Kornek, T. Trittel, U. Strachauer, S. Höme, K. Will, and R. Stannarius, Phys. Rev. Lett. 110, 144102 (2013).

[14] G. Castillo, S. Merminod, E. Falcon, and M. Berhanu, Phys. Rev. E 101, 032903 (2020), and references therein.

[15] E. Falcon, J.-C. Bacri, and C. Laroche, Europhys. Lett. 103, 64004 (2013); Phys. Rev. Fluids 2, 102601(R) (2017).

[16] P. K. Haff, J. Fluid Mech. 134, 401 (1983).

[17] I. Goldhirsch and G. Zanetti, Phys. Rev. Lett. 70, 1619 (1993).

[18] S. E. Esipov and T. Pöschel, J. Stat. Phys. 86, 1385 (1997).

[19] E. Falcon, R. Wunenburger, P. Évesque, S. Fauve, C. Chabot, Y. Garrabos, and D. Beysens, Phys. Rev. Lett. 83, 440 (1999).

[20] S. Aumaître, A. Alastuey, and S. Fauve, Eur. Phys. J. B 54, 263 (2006).

[21] E. Opsomer, F. Ludewig, and N. Vandewalle, Europhys. Lett. 99, 40001 (2012).

[22] M. Noirhomme, F. Ludewig, N. Vandewalle, and E. Opsomer, Phys. Rev. E 95, 022905 (2017).

[23] K. Roeller, J. P. D. Clewett, R. M. Bowley, S. Herminghaus, and M. R. Swift, Phys. Rev. Lett. 107, 048002 (2011).

[24] L. H. Luu, G. Castillo, N. Mujica, and R. Soto, Phys. Rev. E 87, 040202(R) (2013).

[25] D. Puzyrev, K. Harth, T. Trittel, and R. Stannarius, Microgravity Sci. Technol. 32, 897 (2020).

[26] E. Falcon, S. Fauve, and C. Laroche, Eur. Phys. J. B 9, 183 (1999).

[27] M. Heckel, A. Sack, J. E. Kollmer, and T. Pöschel, Phys. Rev. E 91, 062213 (2015).

[28] S. Aumaître et al., Rev. Sci. Instrum. 89, 075103 (2018).

[29] European Space Agency's SpaceGrains project, http://www .spacegrains.org/.

[30] I. F. Sbalzarini and P. Koumoustsakos, J. Struct. Biol. 151, 182 (2005).

[31] J. Schindelin, I. Arganda-Carreras, E. Frise, V. Kaynig, M. Longair, T. Pietzsch, S. Preibisch, C. Rueden, S. Saalfeld, B. Schmid, J.-Y. Tinevez, D. J. White, V. Hartenstein, K. Eliceiri, P. Tomancak, and A. Cardona, Nat. Methods 9, 676 (2012).

[32] M. Noirhomme, A. Cazaubiel, A. Darras, E. Falcon, D. Fischer, Y. Garrabos, C. Lecoutre-Chabot, S. Merminod, E. Opsomer, F. Palencia, J. Schockmel, R. Stannarius, and N. Vandewalle, Europhys. Lett. 123, 14003 (2018).

[33] M. Rovere, D. W. Hermann, and K. Binder, Europhys. Lett. 6, 585 (1988).

[34] M. Rovere, D. W. Hermann, and K. Binder, J. Phys. Condens. Matter 2, 7009 (1990).

[35] L. Onsager, Phys. Rev. 37, 405 (1931).

[36] M. Doi, J. Phys. Condens. Matter 23, 284118 (2011).

[37] See Supplemental Material at http://link.aps.org/ supplemental/10.1103/PhysRevLett.126.128002, which includes Refs. [21,36], for details on the theoretic model.

[38] S. Herminghaus and M. G. Mazza, Soft Matter 13, 898 (2017).

[39] J. P. D. Clewett, J. Wade, R. M. Bowley, S. Herminghaus, M. R. Swift, and M. G. Mazza, Sci. Rep. 6, 28726 (2016). 